\title{
La poterne des Quatre-Mares et son environnement architectural, Sens (Yonne)
}

Jérôme Mercier

\section{(2) OpenEdition}

1 Journals

Édition électronique

URL : https://journals.openedition.org/cem/11180

DOI : $10.4000 /$ cem. 11180

ISSN : 1954-3093

Éditeur

Centre d'études médiévales Saint-Germain d'Auxerre

Édition imprimée

Date de publication : 15 août 2009

Pagination : 29-32

ISSN : 1623-5770

\section{Référence électronique}

Jérôme Mercier, «La poterne des Quatre-Mares et son environnement architectural, Sens (Yonne) », Bulletin du centre d'études médiévales d'Auxerre / BUCEMA [En ligne], 13 | 2009, mis en ligne le 23 septembre 2009, consulté le 22 septembre 2022. URL : http://journals.openedition.org/cem/11180 ; DOI : https://doi.org/10.4000/cem. 11180

Ce document a été généré automatiquement le 22 septembre 2022.

\section{(c)}

Creative Commons - Attribution - Pas d'Utilisation Commerciale - Partage dans les Mêmes Conditions 4.0 International - CC BY-NC-SA 4.0

https://creativecommons.org/licenses/by-nc-sa/4.0/ 


\title{
La poterne des Quatre-Mares et son environnement architectural, Sens (Yonne)
}

\author{
Jérôme Mercier
}

1 La poterne, dite des Quatre-Mares, est située boulevard du Quatorze Juillet. Les observations réalisées dans le cadre d'une étude de bâti ont permis de confirmer son homogénéité générale en élévation. D'après les sources, elle est connue comme étant une construction du XIII ${ }^{e}$ siècle. Une grande phase de construction intervient au début du XIII ${ }^{e}$ siècle afin de mettre en place une porte fortifiée en maçonnerie soignée et décorée d'au moins une peinture à motifs végétaux. Cette construction vient aménager une brèche préexistante dans la courtine gallo-romaine. Elle donne une plus value architecturale et défensive à ce passage et met en communication différents édifices commandés par un notable de la ville de Sens, bailli royal. L'environnement construit, concernant ces bâtiments ainsi que la portion de l'enceinte gallo-romaine, a également pu être abordé lors de cette étude au travers de leurs phases de constructions et de réaménagements postérieurs. Il s'agit d'une opération préliminaire à la restauration de l'édifice. 


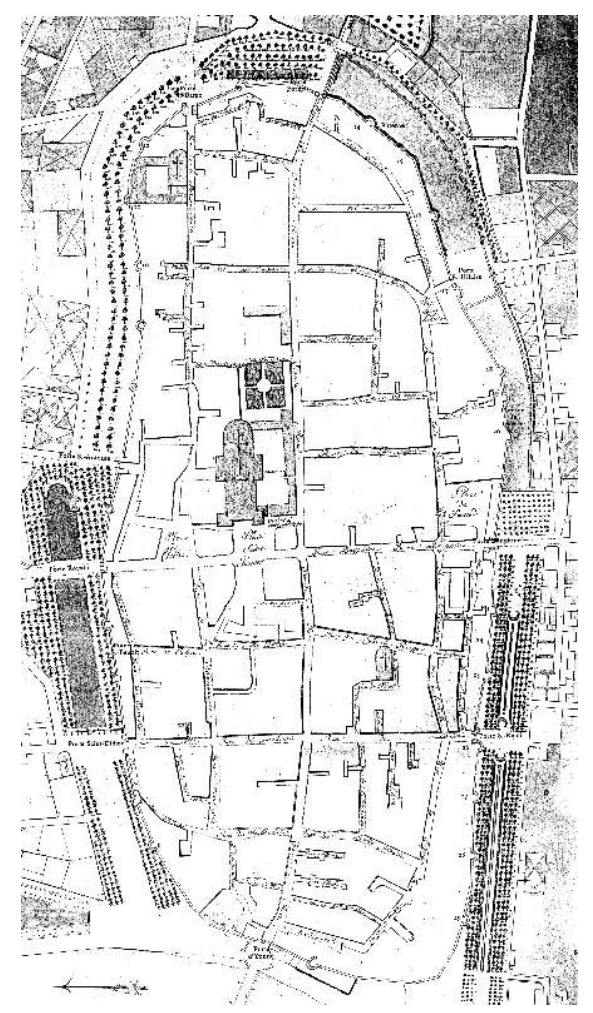

Fig. 1 - Plan de la ville de Sens « fait par Arnoult » en 1805.

2 La ville s'est établie à partir des années 40-50 sur la rive droite de l'Yonne. Entre les années 270 et la fin du IV ${ }^{e}$ siècle, la cité, appelée Senones ou civitas Senonum, connaît une importante transformation avec la construction d'une enceinte fortifiée. Ce castrum, de forme ovale et d'une surface d'environ 40hectares, fait partie des plus vastes enceintes construites en Gaule romaine.

3 La maçonnerie de cette poterne a été réalisée en pierre de Paris. Deux contreforts massifs s'élèvent $\mathrm{du}$ sol et encadrent l'entrée de la ville intra muros. On accède aujourd'hui à la porte par un escalier de trois degrés. Elle est surmontée d'un arc en plein cintre surbaissé. Deux plateformes circulaires, saillantes et soutenues par des corbeaux en retrait, surmontent ces contreforts. Une stèle honorifique antique, jumelle d'une stèle conservée au Musée archéologique de Sens, a été découpée en six parties et deux d'entre elles ont été remployées pour réaliser ces corbeaux.

4 Les blocs employés sont en moyen appareil et les joints, entre eux, sont épais (1 à $2 \mathrm{~cm}$ d'épaisseur) et lissés. Les assises sont régulières et l'ensemble de la construction montre le respect d'un plan d'ensemble, réalisé d'un seul trait.

Deux sections de voûtes surmontent le passage encadré par le massif de la poterne. Elles se situent à deux niveaux différents et bordent les deux rainures parallèles de la glissière d'une herse. Elle court sur toute la hauteur de la poterne. L'intrados de l'arc limitant la glissière présente sur toute sa longueur les vestiges d'une peinture ornementale à motifs de rinceaux végétaux se développant d'est en ouest. Ce décor figure directement sur les claveaux de l'arc et semble contemporain de la construction de la poterne. 


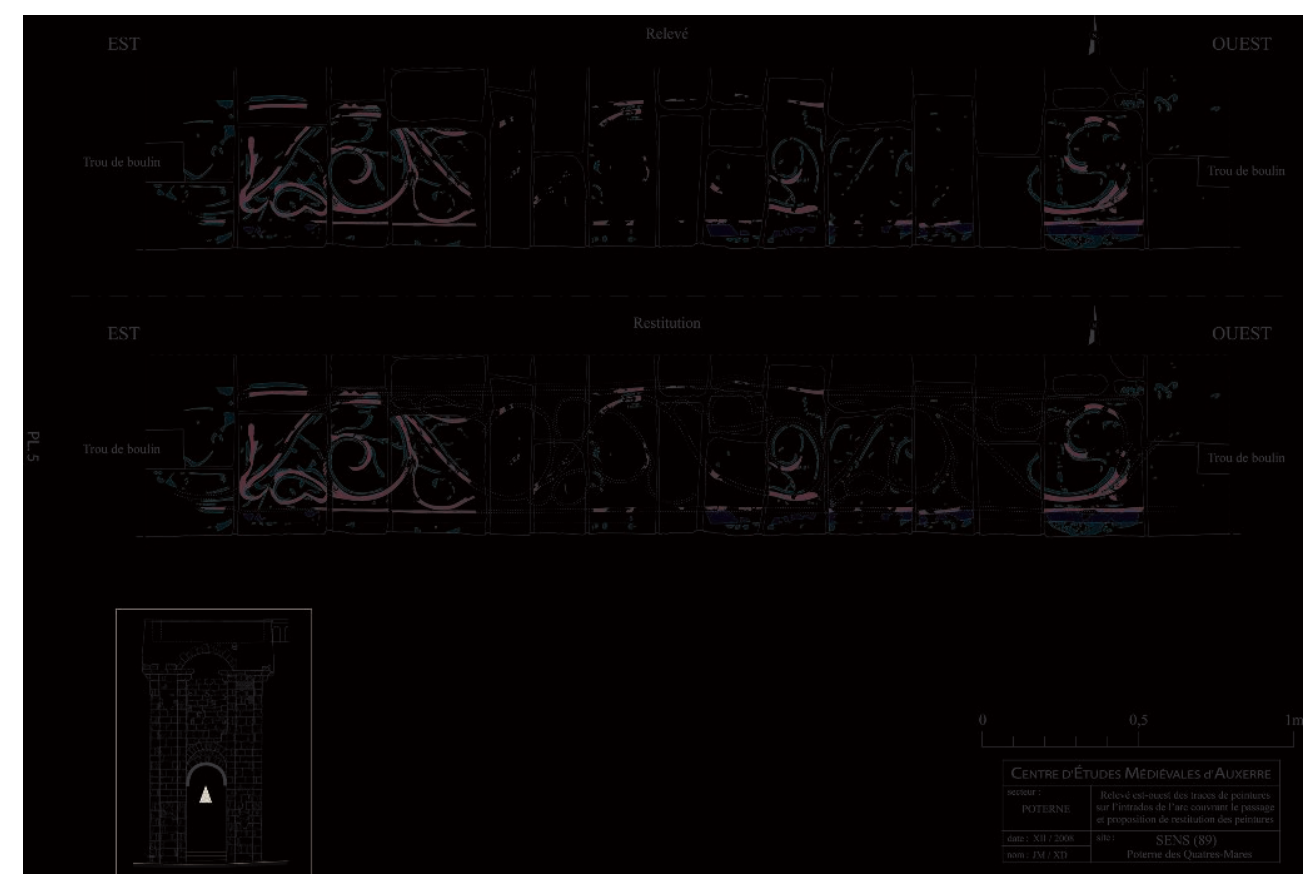

Fig. 2 - Sens. Relevé est-ouest des traces depeintures sur l'intrados de l'arc couvrant le passage. Proposition de restitution (CEM)

6 Sur la terrasse couronnant la courtine, une construction ne comprenant qu'une pièce a été identifiée comme une maison de guet de la fin XVI ${ }^{\mathrm{e}}$-début XVII ${ }^{\mathrm{e}}$ siècle. Son aspect a grandement été altéré depuis, mais des éléments de stratigraphie et sa localisation nous ont conduits à la placer dans l'ensemble défensif utilisé par la milice bourgeoise de Sens sous l'Ancien Régime.

7 Les indices stratigraphiques relevés sont venus renforcer les données existantes dans les sources textuelles et la construction de la poterne est donc bien à situer dans le courant du XIII siècle. Il en est de même pour tout l'ensemble architectural commandité par Garnier des Prés, selon trois phases de chantier proches.

8 L'ensemble construit présente une grande homogénéité. À part dans quelques réparations ponctuelles, il montre des mortiers très proches entre eux. La maçonnerie s'appuie sur une brèche, précédemment aménagée en passage ouvert dans le rempart gallo-romain.

9 Celui-ci montre tous les caractères des canons de construction antique. Ses soubassements sont faits avec des blocs de grand appareil en remplois, sur lesquels est placée une maçonnerie de petit appareil régulier en grès, rythmée toutes les huit à dix assises par des cordons de briques superposées. Le tout est lié grâce à du mortier de tuileau. Deux phases de construction antiques différentes ont été découvertes.

Une dernière phase est venue couronner ce rempart à l'époque médiévale, avant le XIII ${ }^{\mathrm{e}}$ siècle. Nous avons établi que la tour à l'est de la poterne, faisant partie du classement aux Monuments historiques du site et jusque-là considérée comme étant d'époque gallo-romaine, fait en réalité partie de cette même phase de construction. Elle est de plan circulaire et est enchâssée dans le mur d'enceinte. Dans son noyau, nous avons néanmoins mis au jour les traces d'un premier état, inédit jusqu'à maintenant. Il fait partie de la phase initiale de construction antique. 
11 Selon les éléments recueillis lors de cette étude, il apparaît que cet ensemble architectural du XIII ${ }^{\mathrm{e}}$ siècle pourrait être une construction au caractère exceptionnel, en rapport direct avec l'autorité de gouvernement de la ville de son commanditaire. La poterne est une annexe directe de la maison. Elles formaient toutes deux un ensemble architectural majeur de la cité.

12 Cette étude n'ayant pas pour objet l'analyse de la construction de l'ensemble du rempart gallo-romain, nous nous sommes néanmoins intéressés à celle de la courtine située entre la poterne des Quatre-Mares et la tour circulaire immédiatement à l'est. Les éléments observés nous ont conduits à parcourir les portions de l'enceinte encore en élévation, uniquement au sud de la ville, afin de préciser nos premières analyses. Nous en avons conclu qu'il n'était pas raisonnable de voir ce chantier comme une phase de construction extrêmement ordonnée et centralisée.

13 L'origine du tracé « actuel » du rempart gallo-romain n'ayant jamais été complètement élucidée, différents éléments relevés lors de cette étude nous ont amenés à envisager l'hypothèse d'une construction de l'enceinte en deux temps distincts.

14 Parmi ces éléments, l'exhaussement des assises de fondation en grand appareil observé entre la poterne et la tour immédiatement à l'est semble intéressant. Son pendant existe au nord de la ville, également à proximité immédiate d'une autre porte. L'aspect du tracé global de l'enceinte est, de surcroît, singulièrement peu régulier, ceci sans aucune contrainte de relief ou autre.

Ces éléments, et quelques autres, pourraient plaider pour l'hypothèse d'un projet d'enceinte initial dont la superficie aurait été revue à la hausse quelque temps après. Le point de jonction de ces deux projets se trouverait un peu à l'est de notre secteur. Le premier tracé se situerait donc à l'ouest, le long de l'Yonne, revenant jusqu'à quelque distance à l'est du secteur de la poterne. Son tracé aurait donc rejoint, à partir de ce point, le nord de la ville, en passant non loin du chevet actuel de la cathédrale. Les angles dans l'enceinte auraient ainsi été renforcés par l'exhaussement des fondations, afin de supporter les contraintes structurelles induites par le décrochement du tracé.

Le second tracé, augmentant la superficie de la ville close, aurait fait l'objet d'une phase de construction ultérieure, en reprenant les modes de construction employés dans la première partie. La principale différence étant que le matériau employé dans cet agrandissement, dans le petit appareil, n'était plus le grès. La craie, pour une raison de source d'approvisionnement sans doute, est devenue très largement majoritaire. C'est d'ailleurs du petit appareil en craie qui est utilisé dans la reprise de la courtine.

Quant à la datation de ces phases de construction, on sait que la fondation de l'enceinte repose sur des niveaux ruinés à la fin du $\mathrm{III}^{\mathrm{e}}$ siècle. Ces observations se fondent essentiellement sur des niveaux situés à l'ouest, où le tracé d'un cardo secondaire a été détruit par le passage du rempart. Nous avons donc une bonne certitude concernant l'époque de sa construction dans cette partie de la ville, à savoir entre 270 et 300 . À l'est, en revanche, la majeure partie du rempart ayant été démontée au XIX ${ }^{\mathrm{e}}$ siècle, nous ne pouvons pas la dater avec précision. Si l'on admet que deux projets se sont succédé, suivant des méthodes comparables - la préparation du substrat est également similaire dans les deux parties de la ville : des couches de sable et de graviers damées accueillent les blocs de fondations, eux-mêmes encadrés de pieux enfoncés dans le substrat -, il faudrait que ce soit avec un intervalle chronologique relativement faible. L'hypothèse, 
envisagée par certaines études, d'un agrandissement au $V^{e}$ siècle paraît donc trop basse. Il est probable que seules quelques décennies se soient écoulées entre les deux projets. Si ces nouvelles données étaient confirmées, elles permettraient enfin de s'interroger sur la localisation de la cathédrale primitive, encore inconnue, dans l'angle nord-est de l'enceinte.

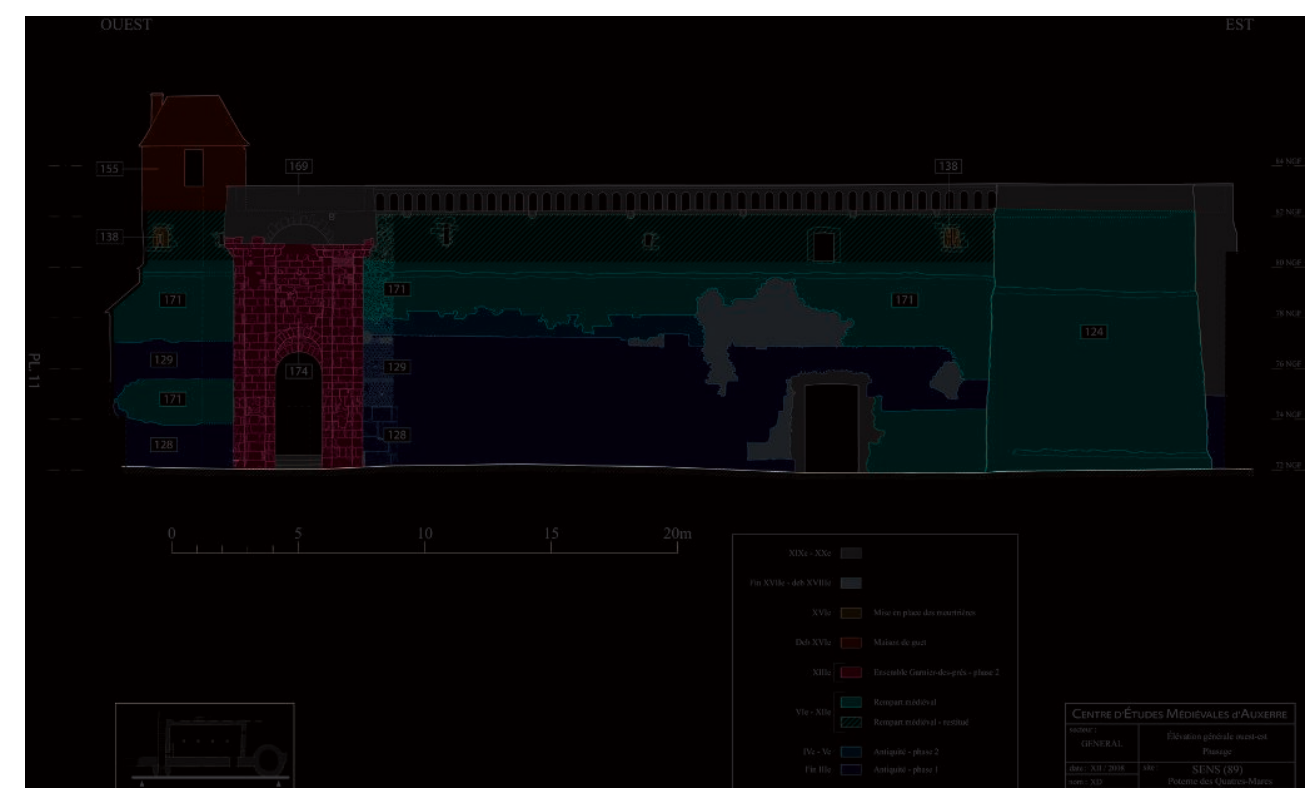

Fig. 3 - Sens. Élévation générale ouest-est - Phasage (CEM).

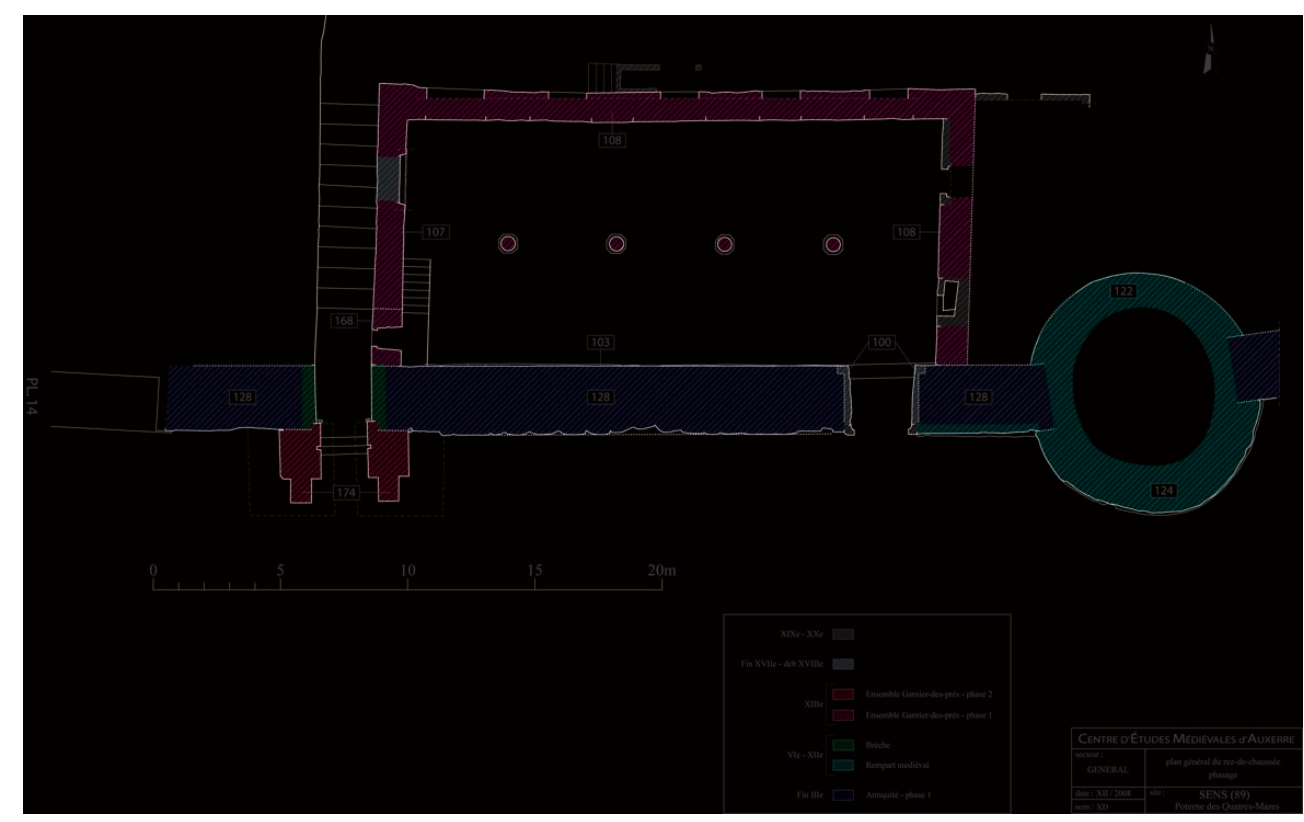

Fig. 4 - Sens. Plan du rez-de-chaussée. Phasage (CEM). 
INDEX

Index géographique : France/Sens

Mots-clés : poterne 